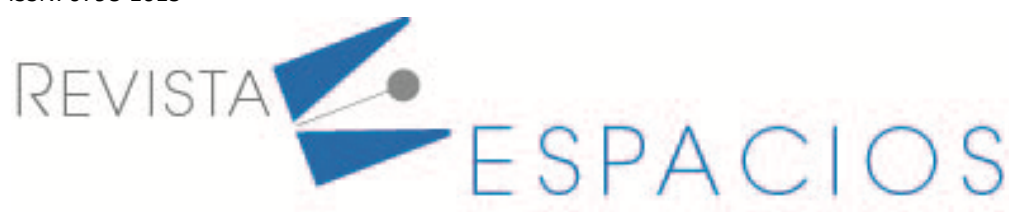

Vol. 41 (49) $2020 \cdot$ Art. 6

Recibido/Received: 29/08/2020 • Aprobado/Approved: 18/11/2020 • Publicado/Published: 23/12/2020

\title{
Análisis administrativo de la implementación de la historia clínica portátil en un hospital de Bucaramanga (Colombia)
}

\section{Administrative analysis of the implementation of portable clinic history in a hospital in Bucaramanga}

\author{
ALMEIDA, Alexander ${ }^{1}$ \\ TORRES, Dagoberto ${ }^{2}$ \\ GALLARDO, Nadeska ${ }^{3}$
}

\begin{abstract}
Resumen
La historia clínica es el documento legal para el manejo de datos en el sector salud. Los episodios de actualización y optimización de esta juegan un papel clave en la prestación final del servicio de salud. Por tal razón se pretende analizar el impacto administrativo de la implementación de un prototipo de lectura y actualización de historia clínica portátil en un hospital de la ciudad de Bucaramanga.

Palabras clave: historia clínica portátil; impacto administrativo; dispositivo tecnológico; calidad en salud
\end{abstract}

\begin{abstract}
The clinical history is the legal document for data management in the health sector. The updating and optimization episodes of this, play a key role in the final provision of the health service. For this reason, it is intended to analyze the administrative impact of the implementation of a prototype for reading and updating the portable medical record in a hospital in the city of Bucaramanga.

Key words: portable medical history; administrative impact; Technological device; quality in health
\end{abstract}

\section{Introducción}

El registro de la información hospitalaria por medio de la historia clínica, toma un papel determinante en el diagnóstico, manejo y rehabilitación final de un cuadro clínico en un paciente que consulta a instituciones prestadoras de servicios de salud (Ajami \& Bagheri-Tadi, 2013); es por esto, que resulta indispensable garantizar un manejo efectivo de la historia clínica y buscar por medio de herramientas tecnológicas, la optimización, mejora y adaptación continua de la misma y así evitar complicaciones o evento adversos en el ámbito clínico institucional (Ynalvez y Shrum, 2014), que pueda alterar el curso normal del manejo hospitalario.

La historia clínica debe ser única, integrada y acumulativa para cada paciente en el hospital, donde debe existir un sistema eficaz de recuperación de la información clínica, que permita su actualización, manejo e interpretación, en pro de dar un sentido eficiente de tratamiento oportuno a un usuario que solicite los servicios

\footnotetext{
${ }^{1}$ Docente Universitario - Investigador. IPRED - R.F. Universidad Industrial de Santander. alexalme@correo.uis.edu.co

2 Docente Universitario - Investigador. Facultad de Ciencias Económicas. Universidad de los Llanos. dtorres@unillanos.edu.co

${ }^{3}$ Docente Universitaria - Investigadora. Facultad de Negocios y Ciencias Empresariales. Unisabaneta. nadeska.gallardo.docente@unisabaneta.edu.co
} 
(Castro y Gámez, 2010). Sin colocar en riesgo la integridad de la información y la funcionalidad de ella en un contexto de tratamiento o intervención clínico - asistencial (Lehmann, 2006)

Así mismo, la principal función de la historia clínica es la de garantizar un óptimo registro de la información consultada, ya que permite ser garante de una atención continuada en los pacientes, brindando una relación de costo - beneficio entre el cuerpo clínico de intervención y el usuario (Guerra \& Laguzzi, 2009); la cual se traduce en mecanismos de calidad y consecución final de un adecuado llenado de historia clínica confiable que favorezca al usuario y su interpretación. A su vez, la historia clínica es una de las formas de registro del acto médico, cuyas cuatro características principales se encuentran involucradas en su elaboración y son: profesionalidad, ejecución típica, objetivo y licitud (Guzmán y Arias, 2012).

Por estas razones, garantizar un espacio de actualización constante y el cambio del esquema del llenado en papel a un registro electrónico y más aún cuando se consigue la portabilidad de la historia clínica, en dispositivos inteligentes y cómodos al usuario, se convierte en una alternativa objetiva, propia de los avances tecnológicos y de la alianza del sector salud con las tecnologías de Información y Comunicación (TICs) (Edwards et al, 2014)

Frente a esto, autores como Cubero et al. (2000), mencionan que la trazabilidad de mejoramiento del papel a los dispositivos electrónicos de registro de información de historia clínica es fundamental en el mundo de hoy, para ser garantes de un buen esquema de atención en salud, la cual va de la mano de la conservación del medio ambiente, la economía a gran escala, el compromiso global económico y el fortalecimiento de la economía naranja en el ámbito hospitalario y social (Rueda y Clausen, 2006)

Sumado a esto, el uso de esta historia clínica y la implementación de un equipo de lectura y actualización, garantizarán de forma inmediata la oportunidad en la atención, la pertinencia en la asignación de tareas específicas sobre su patrón de consulta y la seguridad que emana el proceso mismo de consulta sea por urgencia (Rojas et al, 2015), programada o de cualquier índole al momento de acudir a una institución de salud en el ámbito local y nacional.

Todo esto acompañado de un bosquejo normativo y procedimental asumido por la institución prestadora de servicios de salud o consultorios particulares, en los cuales se encamine el direccionamiento de modelos de atención basados en evidencia científica (Marco \& Salvador, 2017) y en la búsqueda constante de la satisfacción de un usuario que consulta el servicio de salud.

El dispositivo de lectura - actualización de datos de la historia clínica portátil, permite almacenar los datos más relevantes de la misma, en medios de almacenamiento inteligentes y cómodos, donde se garantiza la seguridad en el manejo de la información y permite la consulta remota de los historiales para utilizarlos en casos de emergencia y cualquier tipo de consulta médica general y especializada en un Hospital de la ciudad de Bucaramanga

A su vez, con esta implantación, los profesionales de salud, personal médico y hospitalario contaran con una herramienta inteligente, que suministra oportunamente la información básica sobre la historia clínica de las personas, para que sea utilizada en cualquier sitio o circunstancia, tanto para casos de emergencia como en medicina preventiva o en intervenciones quirúrgicas que se le deban realizar a un paciente.

Sin embargo, la viabilidad de implementación de este dispositivo de lectura- escritura de la historia clínica y su portabilidad, debe ser analizada desde dos puntos de vista; el primero más estratégico a la interpretación del sector clínico - asistencial y el segundo al campo administrativo - financiero, el cual determina a ciencia cierta, si es posible, viable, seguro y genera costo - efectividad, la puesta en marcha de este cambio tecnológico en la historia clínica y el manejo de esta, en el modelo de salud de la entidad hospitalaria de la ciudad de Bucaramanga. 


\subsection{Impactos administrativos y clínicos - asistenciales, de la implementación de la portabilidad de la historia clínica}

La historia clínica portátil y su mecanismo de actualización, toma relevancia en el campo administrativo, para su respectivo análisis y viabilidad de implementación del dispositivo en los servicios consultantes de usuarios y manejo de información clínica y en el aspecto clínico - asistencial, el cual juega un papel determinante en la aprobación o no del contexto de seguridad, pertinencia, oportunidad y manejo de la esta, en el actuar diario clínico de la situación de salud (Lanza, 2005).

Dentro de las ventajas de estructura, encontramos que se mejora el acceso a la información, la recuperación de una pieza específica de la historia es mucho más rápida, diferentes usuarios autorizados pueden consultar la misma información desde distintos puntos y en forma simultánea, a su vez, se facilita la comunicación del equipo de salud y se garantiza el acceso en casos de emergencia (Gil \& Viega, 2018).

Otra ventaja de orden clínico - asistencial y de manejo administrativo, es que solo existe una historia clínica, no hay evidencias físicas, no hay archivos sueltos y esta puede ser sujeta de verificación y revisión por parte del personal sanitario, que intervienen con la atención del usuario y manejo clínico; así mismo, cada paciente tiene así un solo número de historia y su gestión queda centralizada en un archivo único, garantizando que todos los sucesivos episodios de ese paciente queden conservados juntos (Medrano y Pacheco, 2015).

Otra ventaja se da frente a aspectos operativos, económicos y ecológicos (Curioso, 2014). Esta solamente aplica al manejo de historias clínicas de forma digital, donde se aprecia el verdadero valor de este enfoque de ventaja competitiva, ya que en forma fisca la historia clínica, genera una gran cantidad de contaminación y situaciones desfavorables para el medio ambiente, debido a la tala de árboles y fabricación de materia prima para el objetivo mencionado (Indarte, 2016); de igual forma, genera inconvenientes de espacio fisco para el almacenamiento de las mismas. Otra dimensión aplica en la humedad y malos olores que puede generar el acumulo de papel y el costo que conlleva la impresión de todo este tipo de papelería (González y Luna, 2012).

Así mismo, para autores como Plazzotta et al (2015), las ventajas médicas incorporadas en la historia clínica, se dan cuando se logra la integración de imágenes digitales, las cuales pueden ser revisadas a distancia por médicos expertos en aquellas situaciones en las que se lo requiera, facultad que garantiza en u forma inicial, este dispositivo de lecto- escritura y almacenamiento de la historia clínica portátil. A su vez, el ingreso estandarizado de datos y el uso obligatorio de algunos campos para pasar de pantalla disminuye la posibilidad de olvidos y errores, en este inteligente dispositivo mencionado.

Por otro lado, desde el punto de vista administrativo y funcional, permite concentrar sistemas de apoyo a la decisión clínica (algoritmos y protocolos de estudio y tratamiento), recordatorios de práctica y conexión con cuerpos de conocimiento médico. Del mismo modo, cuenta con un respaldo legal - ético, donde se busca que la historia clínica, contribuya a que la documentación médica sea llevada de acuerdo a los requisitos formales establecidos por las distintas normativas (Franco et al, 2007). Esta debe ser legible, no tener espacios en blanco ni enmendaduras, debe tener firma por el profesional tratante. Los campos de fechas y horas deben ser obligatorios, los campos del usuario y datos generales deben estar registrados (Gil \& Viega, 2018).

Otro aspecto de orden administrativo se encaminan a que se reducen los costos y manejos de espacios locativos para impresión, administración, uso, operacionalización (Huerta et al, 2009), custodia y archivo central de la historia clínica en papel, gestionando estos procesos de forma estratégica y más asertiva, con el fin de generar beneficios propios de índole financiero, que puedan apoyar otros proceso claves a nivel institucional.

Sumado a lo anterior, la historia clínica portátil extiende una responsabilidad de orden administrativo-gerencial, debido a que es el documento más importante para respaldar los procedimientos practicados, las complicaciones 
sufridas y los costos monetarios de quienes responden por cada paciente. En el actual sistema de salud colombiano, es el soporte vital para la construcción de una factura y poder recobrar el servicio prestado a una entidad promotora de servicio de salud contratante con la institución (Riffon et al, 2014); todo esto define una ruta crítica de manejo, supervisión, alcance e interpretación, con el fin de ser un garante de prestación del servicio de salud y un apoyo constante y latente en todo el registro y almacenamiento de datos clínicos, en favor de la institución, su público objetivo y su planeación estratégica.

Finalmente, la historia clínica tiene implicaciones médico-legales, esto debido a que entre el paciente y el profesional de salud que lo atiende, se establece la típica relación de contrato consensual, por la cual el profesional se compromete a colocar todos los medios de su parte para devolver o mantener la salud del paciente, siendo de vital importancia el registro y acompañamiento técnico - administrativo, para gar artizar el mismo (Correa, 2015).

\subsection{Características del diseño del dispositivo de portabilidad de historia clínica}

El esquema de diseño y funcionamiento se estableció en la RFID (Radio Frequency IDentification), la cual es una tecnología que incorpora el uso de acoplamiento electromagnético o electrostático en la porción de radiofrecuencia (RF) del espectro electromagnético para identificar de forma única un objeto, animal o persona; RFID está entrando en uso creciente en la industria como una alternativa al código de barras. La ventaja de la RFID es que no requiere el contacto directo o el escaneo de la línea de vista (Want, 2006)

Para Bhuptani \& Moradpour (2005) se menciona que un sistema RFID consta de tres componentes: una antena, un transceptor (a menudo combinados en un lector) y un transpondedor (la etiqueta o TAG). La antena utiliza ondas de radiofrecuencia para transmitir una señal que activa el transpondedor. Cuando se activa, la etiqueta transmite los datos a la antena. Los datos se utilizan para notificar a un controlador lógico programable que debe producirse una acción. Antes de que la RFID pueda entenderse completamente, es esencial comprender cómo se produce la comunicación por radiofrecuencia.

El modo de funcionamiento de los sistemas RFID es simple. La etiqueta RFID, que contiene los datos de identificación del objeto al que se encuentra adherida, genera una señal de radiofrecuencia con dichos datos. Esta señal puede ser captada por un lector RFID, el cual se encarga de leer la información y pasarla en formato digital a la aplicación específica que utiliza RFID.

La comunicación RF (radiofrecuencia) se produce por la transferencia de datos a través de ondas electromagnéticas. Mediante la generación de una onda electromagnética específica en la fuente, su efecto puede ser observado en el receptor lejos de la fuente, permitiendo la identificación del TAG y posteriormente la transmisión de la información allí contenida para su proceso y análisis (Landt, 2005).

Want (2006) hace mención que un TAG RFID, está compuesta por una antena, un transductor inalámbrico y un material encapsulante. Estas etiquetas pueden ser activas o pasivas. Mientras que las etiquetas activas tienen potencia on-chip, las etiquetas pasivas utilizan la potencia inducida por el campo magnético del lector RFID. Por lo tanto, las etiquetas pasivas son más baratas pero con un rango inferior ( $<10 \mathrm{mts})$ y más sensibles a las restricciones regulatorias y ambientales, en comparación con las etiquetas activas.

Así mismo, Un lector de RFID (Ni, Liu, Lau, \& Patil, 2004), consiste en una antena, un transceptor y un decodificador, que envía señales periódicas para investigar sobre cualquier etiqueta que pueda encontrarse dentro de la vecindad. Al recibir cualquier señal de una etiqueta, el lector es capaz de realizar la lectura de la información contenida en el TAG y la transmite al procesador de datos para su respectivo análisis. 
El subsistema de procesamiento de datos proporciona los medios para procesar y almacenar los datos. En la mayoría de los casos, este sistema puede estar soportado en el uso de Microcontroladores, los cuales permiten la implementación de sistemas embebidos de bajo costo orientados a la ejecución de tareas específicas de procesamiento, ejecución y análisis de la información recibida (Elías, 2016)

La banda LF cubre frecuencias de $30 \mathrm{KHz}$ a $300 \mathrm{KHz}$. Por lo general, los sistemas RFID LF operan a $125 \mathrm{KHz}$, aunque hay algunos que operan a $134 \mathrm{KHz}$. Esta banda de frecuencia proporciona un rango de lectura corto de $10 \mathrm{~cm}$ y tiene una velocidad de lectura más lenta que las frecuencias más altas, pero no es muy sensible a la interferencia de ondas de radio.

Existen varios estándares RFID HF, como la norma ISO 15693 para el seguimiento de artículos y las normas ECMA340 e ISO / IEC 18092 para Near Field Communication (NFC), una tecnología shortrange comúnmente utilizada para el intercambio de datos entre dispositivos. Otros estándares de HF incluyen las normas ISO / IEC 14443 A y ISO / IEC 14443 para la tecnología MIFARE, que se utilizan en tarjetas inteligentes y tarjetas de proximidad, y el JIS X 6319-4 para FeliCa, que es un sistema de tarjetas inteligentes comúnmente utilizado en dinero electrónico Tarjetas.

La banda de frecuencia UHF cubre el rango de $300 \mathrm{MHz}$ a $3 \mathrm{GHz}$. Los sistemas que cumplen con la norma UHF Gen2 para RFID Utiliza la banda de 860 a $960 \mathrm{MHz}$. Si bien existe cierta variación en la frecuencia de una región otra, los sistemas UHF Gen2 RFID en la mayoría de los países entre 900 y $915 \mathrm{MHz}$.

A su vez, este dispositivo utilizo aplicaciones TAGs, los cuales pueden ser encontrados comercialmente en forma de tarjetas o llaveros con capacidades de almacenamiento de memoria EEPROM de $1 \mathrm{~Kb}$ y $4 \mathrm{~Kb}$. Este tipo de TAG está conformado por dos elementos electrónicos básicamente: un microcontrolador especializado conocido como 'chip' y una antena para la trasmisión-recepción de información a una frecuencia de 13.56 Mhz, acorde con el estándar empleado en los sistemas RFID (Gibbs \& González, 2017).

El microcontrolador posee una memoria EEPROM con capacidad de lectura y escritura, la cual permite no solo almacenar el número de serie del TAG, conocido como NUID (Non Unique Identification Number) de 4 bytes que es pregrabado de fábrica, así como la información adicional que el usuario desee almacenar. Algunos TAGs fabricados recientemente, cuentan con un número de serie de 7 bytes, denominado UID (Unique Identification Number), el cual está diseñado para aplicaciones de mayor complejidad.

Finalmente, la historia clínica portátil cuenta con una capacidad de almacenamiento y procesamiento lo suficientemente robusta, como para implementar soluciones complejas y con capacidad de articulación con sensores y otros dispositivos a través de sus GPIOs. Su alto grado de integración en el chip permite un mínimo de circuitos externos, incluyendo el módulo de front-end, está diseñado para ocupar un área mínima de PCB. EI ESP8266 admite APSD para aplicaciones VolP e interfaces de coexistencia Bluetooth. Adicionalmente contiene sistema de RF autocalibrado, el cual permite trabajar en todas las condiciones de funcionamiento sin necesidad de elementos adicionales de comunicación (Kotiyal \& Muzamil, 2016)

\section{Metodología}

Se realizó un estudio con diseño descriptivo-explicativo, con un enfoque cuantitativo, donde se extiende en primera instancia en identificar administrativamente, la viabilidad, costo efectividad y alcance de la implementación de la historia clínica portátil como dispositivo tecnológico y seguidamente, la capacidad de aceptación, sensibilización y adaptación del dispositivo en el personal clínico que maneja historia clínica y pacientes, en un hospital de la ciudad de Bucaramanga. 
El diseño de la investigación es transversal, ya que la descripción se hizo en un único momento temporal. En este estudio se recogió información de profesionales y auxiliares de salud, personal administrativo (registro de información, análisis de implementación, operatividad, cálculos de diseño, margen de utilidad y viabilidad) que hacen parte del hospital, interpretando el análisis estratégico claro de comportamiento administrativo y costo efectivo de la implementación.

La recolección de la información se da en un hospital de la ciudad de Bucaramanga, donde los datos proporcionados por la entidad prestadora del servicio de salud (variables referentes a campos de entrada de la historia clínica, software de distribución, datos sociodemográficos, que se registrarán, para luego ser implementados en el prototipo que se diseñara); de igual forma, se realizarán los estudios previos de diseño del dispositivo, la continuidad y forma de empalme con el diseño de la historia clínica portátil para su optimización.

Con esta información recolectada, se analizar la capacidad de uso, adecuación y viabilidad de dispositivo en cuanto a parámetros de costos de interpretación, seguido por el conocimiento, la percepción, uso, aplicabilidad y sensación final de la historia clínica portátil en sus campos de lectura, actualización en la misma institución y en entidades contratadas en su portfolio de red prestadora de servicios de salud establecida.

Frente a lo anterior, se pretende triangular la información con la literatura científica recogida, sumado a la validación de los datos, al diseño del dispositivo de lectura y actualización, el diseño de la portabilidad de la historia clínica, más la experticia del personal de salud y la percepción de los usuarios, para verificar la efectividad del diseño del mismo y su reproducción en diferentes instituciones de salud de la región.

Para el análisis administrativo, se inició un barrido sectorial de comportamientos claves de orden financiero, de costeo y adaptabilidad, el cual tuvo en cuenta aspectos claves de funcionamiento, espacio, adaptabilidad, de aceptación y abordaje de costo- beneficio. En el aspecto clínico - asistencial, se aplicó un instrumento de medición tipo encuesta, donde las variables se agruparon en cuatro (4) apartados así: modelo de registro, optimización de tiempos de llenado, seguridad de la historia y mecanismos de portabilidad, a los cuales se les asignó diferentes enfoques de calificación y retroalimentación. Para tal fin, se recurrió al personal de salud profesional y auxiliar que labora en la institución hospitalaria, en servicios como Urgencias, Medicina Interna, Pediatría y Ginecobstetricia (Tabla 1).

Tabla 1

Personal de Salud por

Servicios a nivel Hospitalario

\begin{tabular}{ccccc} 
Profesiones & Urgencias & Medicina Interna & Pediatría & Ginecobstetricia \\
\hline $\begin{array}{c}\text { Médicos } \\
\text { Especialistas }\end{array}$ & 4 & 1 & 1 & 2 \\
\hline $\begin{array}{c}\text { Médicos } \\
\text { Generales }\end{array}$ & 3 & 3 & 2 & 2 \\
\hline $\begin{array}{c}\text { Jefes de } \\
\text { Enfermería }\end{array}$ & 2 & 2 & 1 & 1 \\
\hline Fisioterapeutas & 1 & 2 & 1 & 1 \\
\hline $\begin{array}{c}\text { Auxiliar de } \\
\text { Enfermería }\end{array}$ & 4 & 3 & 2 & 3 \\
\hline Total & 14 & 11 & 7 & 9
\end{tabular}




\section{Resultados}

El resultado final de la investigación es el diseño del dispositivo y la puesta en marcha de la portabilidad de la historia clínica, donde se favorece la integración de las interdependencias de servicios, la atención con mejor calidad de los usuarios, la comodidad del mismo frente a que no debe llevar documentación y papel al consultar los servicios de salud; de igual forma, frente al manejo administrativo y financiero de la institución repercute de forma positiva esta implementación del dispositivo de historia clínica portátil, ya que genera mitigación de los costos relacionados con la producción final de la historia clínica y su manejo a nivel interinstitucional.

Los resultados en el orden administrativo muestran como la implementación de este dispositivo en cada uno de los consultorios, áreas, servicios y procesos de atención al usuario en consulta y registro de información clínica asistencial, oscila en más de tres mil (3.000) dólares, en cuyo análisis situacional, se necesitan en promedio ochenta (80), dispositivos de lecto - escritura, actualizaron y portabilidad de la historia clínica en el hospital de la ciudad de Bucaramanga.

Además, la central de alimentación de procesos, captura, manejo y actualización de información, debe contar con un espacio locativo suficiente para su funcionamiento y el valor de instalación, sumado al manejo preventivo, correctivo y de producción, oscila en promedio de cuatro mil (4.000) dólares mensuales. Sumado a esto, los costos de funcionamiento y estrategias de seguimiento están representados en los rubros normales de desarrollo institucional, pactados para le manejo inicial de historia clínica, papelería y bodegaje de custodia de las mismas.

Se suma a lo anterior, las jornadas de capacitaciones, orientaciones y sensibilización, las cuales tienen un comportamiento de gasto en promedio de forma mensual de dos mil (2.000) dólares, teniendo en cuenta la cantidad de personas, funcionarios clínicos y administrativos, que laboran en la entidad. Sin embargo, existe ya la designación de un rubro presupuestal mínimo, que es garante de funcionamiento de este tipo de acciones, cuyos factores de asociación y sinergia de trabajo, están relacionados con la plataforma estratégica y el manual de calidad institucional.

Así mismo, los costos de implementación de este dispositivo y actualización del proceso de historia clínica, compite con los rubros presupuestales y el ámbito financiero del actual sistema de historia clínica, versus la optimización del mismo, ya que es superior el gasto representativo global por este concepto, teniendo en cuenta la actual situación de gestión y manejo de la historia clínica convencional.

El dispositivo de historia clínica portátil, generará un cambios situacionales y de estructura clínica objetiva favorable, ya que con este alcance de medición y tabulación de costeos iniciales, se logra una viabilidad sensible de ejecución y adaptabilidad al sistema, lo que permite un cambio significativo en el manejo del actual proceso y genera consecuencias favorables para el medio ambiente y la economía circulante, que exige cambios estratégicos en la economía mundial.

Finalmente, en el aspecto clínico - asistencial y teniendo en cuenta los apartados de caracterización mencionados anteriormente (modelo de registro, optimización de tiempos de llenado, seguridad de la historia y mecanismos de portabilidad); se establece que en categoría de modelo de registro, las mediciones se enfocan de forma satisfactoria en la forma de diligenciamiento del nuevo esquema de historia clínica portátil, las facilidades de llenado, la seguridad frente al doble registro y la particularidad de tiempos de cargue de información más efectivos, todo esto, de la mano de la simplicidad del cargue de información y la capacidad de reconocimiento de los datos que se registran.

En la categoría de optimización de tiempos de llenado, se define de forma significante el reconocimiento de la practicidad y recurrencia efectiva de tiempos de llenado, siendo rápidos los accesos a campos identificados y mecanismos de lectura básicos de identificación. A su vez, los alcances de particularidad y simplicidad del acceso 
al llenado y la verificación de caracteres que muestran renglones cuando existen errores de conceptos y hasta gramaticales.

La categoría de seguridad de la historia va acompañado frente a la no posibilidad de llenados posteriores o cambios de conceptos establecidos en el diligenciamiento de la misma, simplemente se pueden hacer registros únicos y al momento de subir la información se cierra el canal de llenado posterior, únicamente se puede alimentar campos nuevos; si se debe modificar algún concepto o características se debe hacer bajo consentimiento del usuario y se aclara en la misma su solicitud de cambio, mediante formatos definidos, dentro del mismo sistema y cuyo registro queda identificado en el material de la misma.

En la última categoría, la portabilidad es lo más esencial y favorable, debido a que se evita el usos de papel en físico, la posibilidad de pérdida o daño de la historia clínica convencional, la incomodidad del usuario y los espacios grandes de conservación de la historia clínica en la institución como resguardo y administración; todo esto, mejorado y optimizado con este sistema de portabilidad, debido a que con una sola tarjeta o dispositivo inteligente de poco espacio y características personalizadas, se convierte en una idea práctica, sostenible y amigable al medio ambiente, la cual evita el volumen y el número de documentos que no favorecen la comodidad del usuario y por ende la facilidad y accesibilidad manifiesta, frente al acceso del mismo a la consulta de algún tipo de servicio clínico en la institución.

\section{Conclusiones}

Como novedad y aprovechamiento de las TICs, se logra el diseño e implementación inicial de un sistema de portabilidad de historia clínica institucional, el cual define su alcance en ser un dispositivo practico, de fácil uso, económicamente accesible, el cual puede ser reproducido sin ningún problema; del mismo modo, sirve como mecanismos de registro, seguimiento y monitoreo total del paciente frente a un momento de consulta de salud en cualquier institución que tenga el lector de la misma de forma práctica. Seguido a esto, su implementación generará mecanismos alternativos de índole tecnológico, útiles para el seguimiento de pacientes que pueden ser volátiles o de difícil manejo clínico en un evento de solicitud de servicios clínicos, que puedan ser objeto de desarrollo en el ámbito social, laboral o de la vida cotidiana.

También, este mecanismo de historia clínica portátil impacta favorablemente, en actores de grupos especiales de seguimiento como niños, adultos mayores, mujeres en estado de gestación, personas con discapacidades físicas - mentales y hasta en animales domésticos; todo esto acompañado de un sistema de verificación y lectura eficaces que permita el uso adecuado del dispositivo.

Se evidencia un impacto significativo en la costó-efectividad de la utilización de una historia clínica portátil la cual pueda ser leída, actualizada y codificada en los distintos procesos de atención y consulta de la institución hospitalaria de la ciudad de Bucaramanga seleccionada para tal fin; de igual forma, proporciona información relevante a instituciones de salud (prestadoras - Aseguradoras), donde se dan a conocer las ventajas, oportunidades y lo indispensable de llevar consigo la historia clínica de forma portátil y su actualización continua en puntos clave de lectura.

Así mismo, con esta implementación del dispositivo de portabilidad de la historia clínica, se logra optimizar la utilización de la misma, el manejo informativo, eficacia del registro y demás parámetros útiles para la intervención y registro de cuadros clínicos de salud, logrando la pertinencia y seguridad de atención de los usuarios objeto de este mecanismo de registro de salud institucional.

También se logra implementar un sistema que permite almacenar los historiales clínicos de manera eficiente y económica por medio de tarjetas inteligentes, siendo la portabilidad el valor esencial y fundamental de este 
avance; el sistema desarrollado está conformado por un dispositivo lector-escritor, un portal en Internet y un software de visualización y actualización.

Los resultados obtenidos son satisfactorios, pues se lograron diseñar y construir todos los prototipos necesarios para poner en funcionamiento el sistema. El costo de los prototipos es relativamente económico, comparado con el actual sistema tradicional de historia clínica, lo cual afirma que es una estrategia optativa y sensible de las TICs en todos los aspectos: médico, técnico, tecnológico, administrativo, social y primordialmente financiero.

El análisis administrativo es claro, se obtiene una viabilidad medida en cuanto a costos, operatividad, manejo, espacios, rubros de consecución, permisos de ejecución y diseño que son más efectivos y resultan más satisfactorios frente a un manejo convencional de la historia clínica que se da hoy en día; a su vez, todo el trascender y sensibilización de aplicación de este dispositivo electrónico, genera una expectativa de manejo y resulta coherente con la política institucional hospitalaria y con la puesta en marcha de estrategias efectivas que favorecen una economía circulante y un cuidado del medio ambiente a nivel país.

Por otro lado, se da el visto bueno y condiciones de significativa de sensibilización y aceptación, por parte de los profesionales de salud responsables del diligenciamiento de esta historia clínica portátil, en cuyo ejercicio extienden una serie de contextos favorables, que hacen que esta misma, guarde relevancia de uso y empoderamiento, por parte de todo el personal que tiene que ver con esta prestación de servicios clínicos.

Por último, todo el contexto administrativo, financiero y costo - efectivo es favorable y guarda significancia de asociación con líneas de manejo presupuestal, indicadores de calidad y financieros, que amparadas en un cambio situacional, genera más eslabones de rentabilidad, mitigación de factores de riesgo y perdurabilidad económica, acompañada de una idea interesante de uso de TICs, comparada con la antigua y desgastada historia clínica convencional que existe hoy en día.

\section{Referencias Bibliográficas}

Ajami, S. \& Bagheri-Tadi, T. Barriers for Adopting Electronic Health Records (EHRs) by Physicians. Acta Inform Med. 2013; 21(2):129-34. doi: 10.5455/aim.2013.21.129-134.

https://www.ncbi.nlm.nih.gov/pubmed/24058254

Bhuptani, M., \& Moradpour, S. (2005). RFID field guide: deploying radio frequency identification systems. Retrieved from http://dl.acm.org/citation.cfm?id=1076275

Castro I. y Gámez M. Historia clínica. Farmacia Hospitalaria; 2010. pág. 295- 305. Colombia. https://www.sefh.es/bibliotecavirtual/fhtomo1/cap22.pdf

Correa, C. (2015). La historia clínica. Aspectos jurídicos y dilemas en el derecho español y colombiano. Vlel / Vol. 10, N. ${ }^{\circ} 2$ / julio-diciembre / Bogotá, D. C. / Universidad Santo Tomás / pp. 125-144. https://dialnet.unirioja.es/descarga/articulo/6132856.pdf.

Cubero, P. et al. (2000). Uso de un modelo de historia clínica portátil en atención primaria para el seguimiento de los ancianos desplazados rotatorios. Atención Primaria. Vol. 26. Núm. 3. Julio-agosto. https://www.elsevier.es/es-revista-atencion-primaria-27-articulo-uso-un-modelo-historia-clinicaS021265670078632X

Curioso Y. eSalud en Perú: implementación de políticas para el fortalecimiento de sistemas de información en salud. Rev Panam Salud Pública. 2014;35(5/6):437-41.

https://webcache.googleusercontent.com/search?q=cache:baGzhjRmaQkJ:https://scielosp.org/pdf/rpsp/v $35 n 5-6 / 20 . p d f+\& c d=1 \& h l=e s \& c t=c l n k \& g l=c o$ 
Edwards ST, Neri PM, Volk LA, Schiff GD, Bates DW. Association of note quality and quality of care: A crosssectional study. BMJ Qual Saf 2014, May; 23(5):406-13. https://www.ncbi.nlm.nih.gov/pubmed/24287259

Elías, J. C. (2016). Implementación de WPS en el firmware NodeMCU para el ESP8266. Retrieved from https://idus.us.es/xmlui/handle/11441/44574

Gibbs, A., \& González, E. (2017). Implementación de un sistema de control de entrada y salida empleando el módulo de lectura RFID con la tecnología Arduino. Revista de Iniciación Científica. Retrieved from http://revistas.utp.ac.pa/index.php/ric/article/view/1245

Gil J. \& Viega M. Historia clínica electrónica: confidencialidad y privacidad de los datos clínicos. Rev Méd Urug 2018; 34(4):228-233. http://www.rmu.org.uy/revista/proximo/rmu34-4_921-gil-historia.pdf

Gil, J. \& Viega, M. (2018). Historia clínica electrónica: confidencialidad y privacidad de los datos clínicos. Rev Méd Urug; 34(4):228-233. http://www.scielo.edu.uy/pdf/rmu/v34n4/1688-0390-rmu-34-04-102.pdf

González F y Luna D. La Historia clínica electrónica En: Manual de Salud Electrónica Publicación de Naciones Unidas. Enero 2012 LC/L3446. Disponible en www.cepal.org.uy

Guerra J. \& Laguzzi P. La firma digital y la historia clínica web enabled. Gibba \& Biocom. The Biocomputer Research Group of Argentina- Sociedad Argentina de Informática y Salud; 2009. http://www.nobleseguros.com/ARTICULOS_NOBLE/39.pdf

Guzmán F. y Arias C. La historia clínica: elemento fundamental del acto médico. Rev Colomb Cir. 2012; 27: 1524. http://www.scielo.org.co/pdf/rcci/v27n1/v27n1a2.pdf

Huerta, J., Villar, C.; Cuenca, G., Cabronero, F. y Alfaro, M. (2009). El sistema historia clínica digital del sistema nacional de salud- accesibilidad y protección de la información como elementos clave. Volumen 18, Número 1.Enero-Junio. https://webcache.googleusercontent.com/search?q=cache:9aHgGnCGk_UJ:https://dialnet.unirioja.es/des carga/articulo/3016241. pdf+\&cd=22\&hl=es\&ct=clnk\&gl=co

Indarte, S. (2016). La Historia Clínica Electrónica - su impacto en la atención médica. Tendencias en Medicina Año XXV № 49: 42-45. http://tendenciasenmedicina.com/Imagenes/imagenes49/art_06.pdf

Kotiyal, B., \& Muzamil, M. (2016). Home automation using arduino WiFi module ESP8266. Retrieved from http://www.aiktcdspace.org:8080/jspui/handle/123456789/1558

Landt, J. (2005). The history of RFID. IEEE Potentials. Retrieved from http://ieeexplore.ieee.org/abstract/document/1549751/

Lanza, J. (2005). La historia clínica electrónica: ideas, experiencias y reflexiones. Acimed; 13(5). Disponible en: http://bvs.sld.cu/revistas/aci/vol13_5_05/aci02505.htm

Lehmann, D. (2006). Application of information technology in clinical diabetes care. Part 1. Databases, algorithms and decision support. Medical Informatics; 21: 255-378. Disponible en: http://www.2aida.org/aida/mif221-a.htm

Marco G. \& Salvador Y. Representación del conocimiento en historia clínica electrónica: el caso de la Historia Clínica Digital del Sistema Nacional de Salud de España. Scire. 23:1. (en.-jun. 2017) 25-38. ISSN 1135-3716. https://webcache.googleusercontent.com/search?q=cache:wrsB3pFU_AJ:https://www.ibersid.eu/ojs/ind ex.php/scire/article/view/4306/3874+\&cd=1\&hl=es\&ct=clnk\&gl=co 
Medrano J. y Pacheco L. Historia clínica electrónica y confidencialidad. Rev. Asoc. Esp. Neuropsiq., 2015; 35

(126), 249-253. http://scielo.isciii.es/scielo.php?script=sci_arttext\&pid=S0211-57352015000200001

Ni, L., Liu, Y., Lau, Y., \& Patil, A. (2004). LANDMARC: indoor location sensing using active RFID. Wireless Networks. Retrieved from http://dl.acm.org/citation.cfm?id=1035686

Plazzotta F, Luna D, González F. Sistemas de información en salud: integrando datos clínicos en diferentes escenarios y usuarios. Rev Peru Med Exp Salud Pública 2015; 32(2): 343-51. http://www.scielo.org.pe/pdf/rins/v32n2/a20v32n2.pdf

Riffon N, Kerdelhué G, Soualmi LF, Merabti T, Grosjean J, Lamy JB, et al. Evaluating alignment quality between iconic language and reference terminologies using similarity metrics. BMC Med Inform Decis Mak 2014; 14:17. https://bmcmedinformdecismak.biomedcentral.com/articles/10.1186/1472-6947-14-17

Rojas C. Medina C. y Vargas J. "National registry of electronic health records in Peru", Revista Peruana de Medicina Experimental y Salud Pública, vol. 32, n.o 2, pp. 395-396, jun. 2015. https://www.researchgate.net/publication/323943491_Historia_Clinica_Digital_en_Latinoamerica_una_m irada_desde_la_bibliometria

Rueda C. y Clausen P. La historia clínica informatizada. Evaluación de los casos colombiano y español. MedUNAB. Vol. 9 Número 1 - Abril de 2006. https://webcache.googleusercontent.com/search?q=cache:7XNQalLW69sJ:https://revistas.unab.edu.co/in dex.php/medunab/article/download/170/155/+\&cd=1\&hl=es\&ct=clnk\&gl=co

Want, R. (2006). An introduction to RFID technology. IEEE Pervasive Computing. Retrieved from http://ieeexplore.ieee.org/abstract/document/1593568/

Ynalvez M. y Shrum W. "Professional networks, scientific collaboration, and publication productivity in resource constrained research institutions in a developing country", Res Policy, vol. 40, n.o 2, pp. 204-16, 2014. https://journals. plos.org/plosone/article?id=10.1371/journal. pone. 0161281

Esta obra está bajo una Licencia Creative Commons Attribución-NoCommercial 4.0 International

\section{(cc) BY-NC}

